\title{
Chronic non-bacterial osteomyelitis in the jaw
}

\author{
Soung Min Kim ${ }^{1,2}$, Suk Keun Lee ${ }^{3}$ \\ ${ }^{\prime}$ Oral and Maxillofacial Microvascular Reconstruction LAB, Brong Ahafo Regional Hospital, Sunyani, Ghana, \\ ${ }^{2}$ Department of Oral and Maxillofacial Surgery, School of Dentistry and Dental Research Institute, Seoul National University, Seoul, \\ ${ }^{3}$ Department of Oral Pathology, College of Dentistry and Institute of Oral Science, Gangneung-Wonju National University, Gangneung, \\ Korea
}

\begin{abstract}
J Korean Assoc Oral Maxillofac Surg 2019;45:68-75)
Chronic recurrent multifocal osteomyelitis (CRMO) is one of the most severe form of chronic non-bacterial osteomyelitis (CNO), which could result in bone and related tissue damage. This autoinflammatory bone disorder (ABD) is very difficult for its clinical diagnosis because of no diagnostic criteria or biomarkers. CRMO in the jaw must be suspected in the differential diagnosis of chronic and recurrent bone pain in the jaw, and a bone biopsy should be considered in chronic and relapsing bone pain with swelling that is unresponsive to treatment. The early diagnosis of CRMO in the jaw will prevent unnecessary and prolonged antibiotic usage or unnecessary surgical intervention. The updated researches for the identification of genetic and molecular alterations in $\mathrm{CNO} / \mathrm{CRMO}$ should be studied more for its correct pathophysiological causes and proper treatment guidelines. Although our trial consisted of reporting items from Preferred Reporting Items for Systematic Reviews and Meta-Analyses (PRISMA), there are very few articles of randomized controlled trials. This article was summarized based on the author's diverse clinical experiences. This paper reviews the clinical presentation of $\mathrm{CNO} / \mathrm{CRMO}$ with its own pathogenesis, epidemiology, recent research studies, and general medications. Treatment and monitoring of the jaw are essential for the clear diagnosis and management of $\mathrm{CNO} / \mathrm{CRMO}$ patients in the field of dentistry and maxillofacial surgery.
\end{abstract}

Key words: Autoinflammatory bone disorder, Chronic non-bacterial osteomyelitis, Chronic recurrent multifocal osteomyelitis, Chronic recurrent multifocal osteomyelitis in the jaw

[paper submitted 2018. 7. 17 / revised 2018. 8. 13 / accepted 2018. 8. 21]

\section{Introduction}

Autoinflammatory disorders are known as the hyperactivation of the innate immune system without high-titter autoantibodies and autoreactive lymphocytes. Majeed syndrome, deficiency of interleukin-1 receptor antagonist (DIRA), and pyogenic arthritis, pyoderma gangrenosum, and acne (PAPA) syndrome, were the representative autoinflammatory diseases with non-infectious osteomyelitis. Chronic non-bacterial osteomyelitis (CNO) is a kind of an autoinflammatory bone disorder (ABD) and a rare non-infectious inflammatory bone

\section{Suk Keun Lee}

Department of Oral Pathology, College of Dentistry, Gangneung-Wonju National University, 7 Jukheon-gil, Gangneung 25457, Korea

TEL: +82-33-640-2228 FAX: +82-33-642-6410

E-mail:sukkeunlee@hanmail.net

ORCID: https://orcid.org/0000-0001-6255-4197

(c) This is an open-access article distributed under the terms of the Creative Commons Attribution Non-Commercial License (http://creativecommons.org/ licenses/by-nc/4.0/), which permits unrestricted non-commercial use, distribution, and reproduction in any medium, provided the original work is properly cited. Copyright (C) 2019 The Korean Association of Oral and Maxillofacial Surgeons. All rights reserved. disease of unknown etiology that mostly affects children and adolescents ${ }^{1,2}$. Chronic recurrent multifocal osteomyelitis (CRMO) is another diagnostic name for $\mathrm{CNO}$ because of its auto-inflammatory, non-infectious disorder of the bone with recurrent episodes of pain and fever resembling bacterial osteomyelitis with sterile lesion cultures that are unresponsive to antibiotic or anti-inflammatory medications. $\mathrm{CNO} / \mathrm{CRMO}$ was first reported in 1972 by Giedion et al. ${ }^{3}$ as subacute and chronic 'symmetrical' osteomyelitis. CRMO mainly occurred in the children with a female predominance, with the average age of 10 years between the ages of 4 to 14 years. The affected sites are the metaphysis of long bones, pelvis, clavicle, and mandible ${ }^{1-5}$.

$\mathrm{CNO}$ can appear at any site in the skeleton exhibiting radiographic similarities to bacterial osteomyelitis with osteolytic and sclerotic lesions. Due to the sterile inflammation of the involved bone and atypical clinical features, the culture of the affected sites shows no bacterial infections. Only a biopsy should be considered for the final diagnosis of $\mathrm{CNO}$ to exclude both infected osteomyelitis and malignant bone disease. 
Table 1. Comparisons between chronic recurrent multifocal osteomyelitis (CRMO) and infectious osteomyelitis in jaw

\begin{tabular}{|c|c|c|}
\hline & CRMO & Infectious osteomyelitis \\
\hline Age of onset (yr) & 10 & $<5$ \\
\hline Affected bones & Several & One \\
\hline $\begin{array}{l}\text { Personal or familial } \\
\text { history }\end{array}$ & Psoriasis or IBD & None \\
\hline $\begin{array}{l}\text { Bacterial culture from } \\
\text { the bone or blood }\end{array}$ & Negative & Positive \\
\hline Recurrence & Common & Rare \\
\hline Management & Medication & Surgery+medication \\
\hline Biomarkers & No revealed yet & Inflammatory markers \\
\hline Follow-up & Before adolescent & Until healing periods \\
\hline
\end{tabular}

(IBD: inflammatory bowel disease)

Soung Min Kim et al: Chronic non-bacterial osteomyelitis in the jaw. J Korean Assoc Oral Maxillofac Surg 2019

(Table 1) This review article will reveal the basic nature of $\mathrm{CNO} / \mathrm{CRMO}$ with a specific focus on the jaw for a better understanding and ideal management of ABD by maxillofacial surgeons.

\section{Clinical Presentation of CNO/CRMO in the Jaw}

The clinical presentation of $\mathrm{CNO} / \mathrm{CRMO}$ in the jaw is very diverse with the main symptom of recurrent jaw pain and swelling with or without a general fever. Localized skin redness caused by paraosseous inflammation is a rare symptom. Inflammatory laboratory markers including erythrocyte sedimentation rate (ESR), absolute neutrophil count and white blood cell count (WBC), could be increased. These first findings in an outclinic setting could allow clinicians to suspect any kind of bacterial osteomyelitis in the jaw. CRMO in the jaw commonly has symmetrical and multifocal bony lesions in the long bones exhibiting a lytic lesion or progression to sclerosis or hyperostosis radiographically ${ }^{6,7}$. Unlike other inflammatory osteomyelitis, CRMO has no infectious sources such as retained roots, periodontitis, or pathology in the oral cavity. No previous bisphosphonate therapy or radiation therapy could exclude bisphosphonate related osteonecrosis of jaw (BRONJ) or osteoradionecrosis. A bone biopsy is required to confirm the final diagnosis and to exclude any infected bacterial osteomyelitis or other malignancies. Even though the histopathological features are non-specific for CRMO, most of the bone biopsy could show slight inflammation with lymphocyte infiltration with fibrosis and lack of organisms.

In adult CRMO cases, most of the patients could be usually diagnosed with complex symptoms of synovitis, acne, pustu-

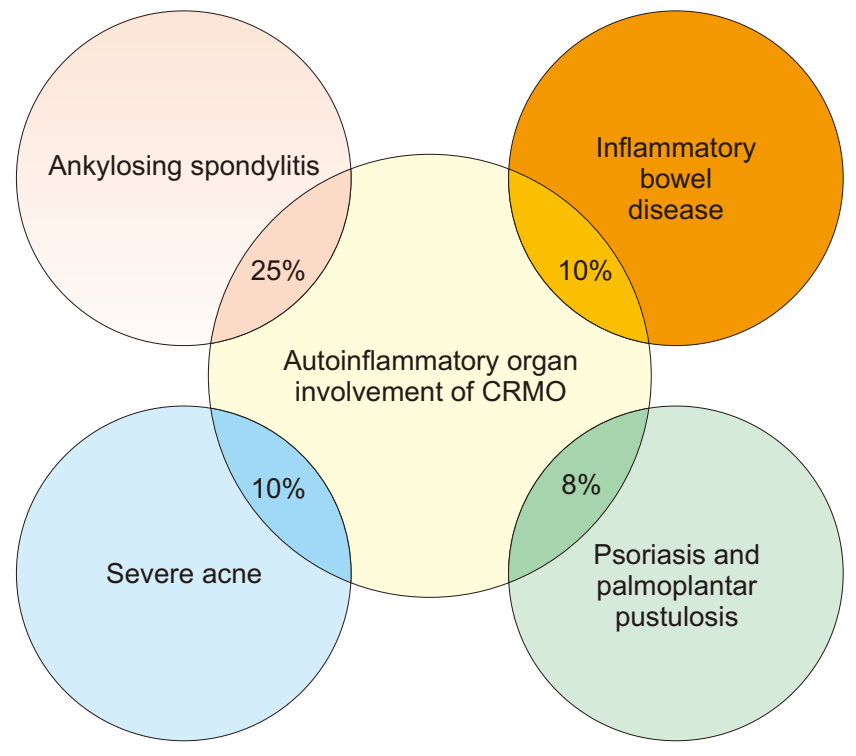

Fig. 1. Schematic drawing showing the autoinflammatory organ involvement of chronic recurrent multifocal osteomyelitis $(\mathrm{CRMO})^{8,9}$.

Soung Min Kim et al: Chronic non-bacterial osteomyelitis in the jaw. J Korean Assoc Oral Maxillofac Surg 2019

losis, hyperostosis, and osteitis (SAPHO), which is currently common with additional symptoms in the Western region. Autoinflammatory organ involvement in CRMO is seen in $8 \%$ of cases with psoriasis and palmoplantar pustulosis, $10 \%$ with inflammatory bowel disease (IBD), 10\% with severe acne, and $25 \%$ with ankylosing spondylitis ${ }^{8,9}$.(Fig. 1)

\section{Epidemiology and diagnosis}

Epidemiological data in $\mathrm{CNO} / \mathrm{CRMO}$ is scarce and even more rare with jaw involvement data compared to other bony involvements from simple case reports, case series, and regional cohort studies. Although there have been no global epidemiologic studies until now, the greatest incidence of sporadic CNO has been found in European countries. Genetic predispositions could be suggested in the pathophysiology of sporadic CNO from familial clusters of CNO/CRMO and high incidences of comorbid-affiliated inflammatory conditions including psoriasis and IBD in CNO patients and firstdegree family members by approximately $50 \%{ }^{10,11}$.

$\mathrm{CNO}$ is occurred in all ages with 7 to 12 years peak onset, female predominant. The clinical presentation of CNO is not typical having a relative self-limiting monofocal bone lesions or the most severe form of CRMO. Clinical signs of $\mathrm{CNO}$ / CRMO include bone pains having arthritis, local swelling with heat and redness. And associated skin manifestations could involve palmoplantar pustulosis, psoriasis, and acne. 
Elevated temperatures or pathological fractures of affected vertebra could be occurred in severe cases ${ }^{10,12}$.

Asymptomatic bone lesions occur on the metaphysis of long bones, clavicle, humerus, vertebral column, pelvic region, femur, tibia, ankle, and calcaneus. Due to these clinical signs, CNO/CRMO could sometimes be mistaken as infectious osteomyelitis secondary to a rather mild type. Routine laboratory findings with inflammatory parameters such as WBC, C-reactive protein (CRP), and ESR, are usually normal or mildly elevated.

Radiographic imaging is essential for $\mathrm{CNO} / \mathrm{CRMO}$ diagnosis with excluding differential diagnoses. Inflammatory bony lesions as radiolucent or sclerotic lesions in plain radiographs could be identified, and computed tomography or magnetic resonance imaging (MRI) are helpful tools particularly in early stage of $\mathrm{CNO} / \mathrm{CRMO}^{13,14}$, and especially in CRMO in jaw.(Fig. 2) MRIs could detect initial bony inflammation with surrounding soft tissue edema in the T2-weighted sequences and gadolinium-enhanced T1 sequences with fat saturation. Whole body MRI imaging could be done for checking any silent lesions, especially in the vertebral column, and for monitoring of disease occurrences such as linear fractures or surrounding soft tissue damage during the follow-up period ${ }^{15,16}$.

Bone biopsies for the definite and differential diagnosis of $\mathrm{CNO} / \mathrm{CRMO}$ should be performed, and any suspicious disease under 2 years of age should be followed by a prompt bony biopsy, due to its rarity ${ }^{17,18}$. Bony malignancies including lymphoma, leukemia, and primary and secondary bone tumors, could be diagnosed with other infectious diseases and immune diseases such as immunodeficiency in interleukin (IL)-12, Langerhans cell histiocytosis (LCH), and other autoinflammatory disorders and should be diagnosed dif- ferentially ${ }^{17,18}$. Cellular atypism from innate immune cells to leukocytes and plasma cells could suggest a disease course between innate and adaptive immune mechanisms ${ }^{19,20}$.

\section{Sporadic CNO/CRMO}

Sporadic CNO/CRMO has been known to have a broad clinical symptoms from mild monofocal bone inflammation to severe recurrent multifocal inflammation, such as vertebral compression fracture, arthritis, skin, or intestinal inflammatory disorder. This sporadic CNO/CRMO could be presented as clinically related phenotypes regardless of envirionmental factors. Most of sporadic type occurred in childhood or preadolescent ages between 7 to 12 years of age, additional bony inflammation could also be occurred later, but this is not severe compared with monogenic $\mathrm{CNO} / \mathrm{CRMO}^{2,21}$.

The pathophysiological understanding of sporadic $\mathrm{CNO} /$ CRMO is less known as the imbalance between proinflammtory and antiinflammatory cytokines, of which the mechanisms are known to govern the cell and receptor-specific induction of cytokines and chemokines ${ }^{22,23}$. Monocytes from CRMO patients fail to express the immune regulatory IL10 in response to Toll-like receptor (TLR) 4 stimulation with lipopolysaccharide. Impaired IL-10 expression is partially caused by the reduced activation of mitogen-activated protein kinases (MAPK) and extracellular signal regulated kinase (ERK) 1, resulting in impaired activation and nuclear shuttling of the transcription factor signaling protein (SP) 1 and subsequent altered recruitment of Sp-1 to the IL-10 promot$\mathrm{er}^{24,25}$.
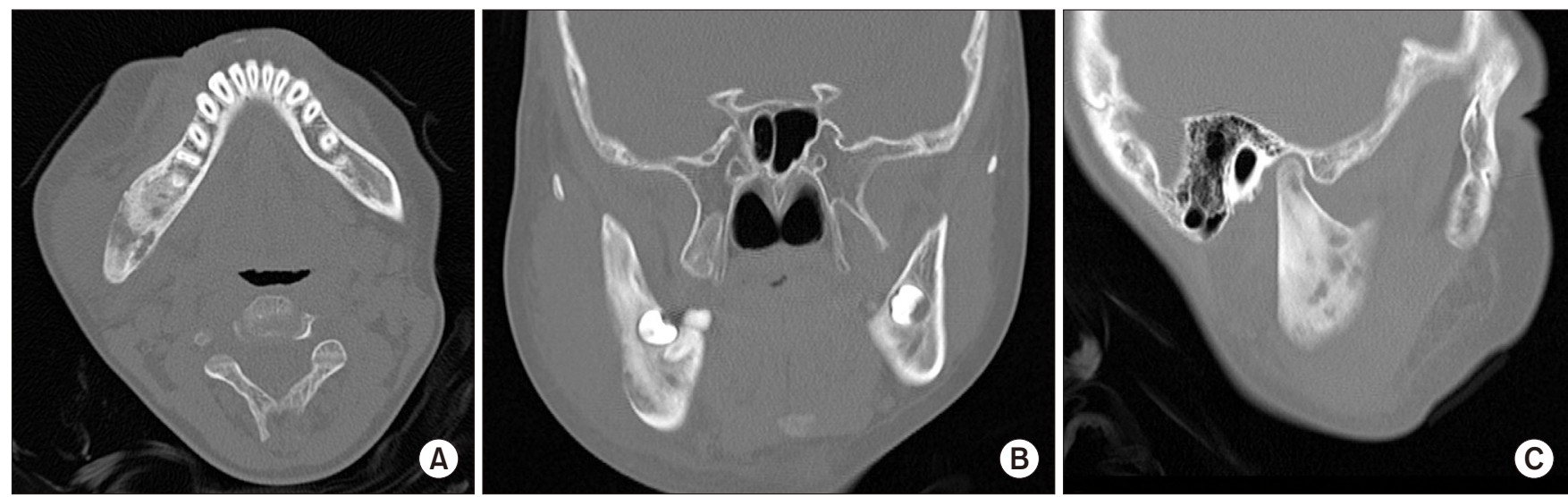

Fig. 2. Representative computed tomographic views of chronic recurrent multifocal osteomyelitis in jaw case showing the lesion in the right mandible, axial (A), coronal (B), and sagittal (C).

Soung Min Kim et al: Chronic non-bacterial osteomyelitis in the jaw. J Korean Assoc Oral Maxillofac Surg 2019 


\section{Familial or monogenic $\mathrm{CNO} / \mathrm{CRMO}$}

Familial or monogenic CNO/CRMO is not known its molecular pathophysiology, but single gene mutations involving $\mathrm{CNO} / \mathrm{CRMO}$ are known to three main diseases such as Majeed syndrome by LPIN2 mutations, DIRA by IL1RN mutations, and PAPA by proline-serine-threonine phosphataseinteracting protein 1 (PSTPIP1) mutations. These monogenic disorders are associated with multiprotein complexes, inflammasomes which mediate the cleavage and subsequent release of proinflammatory cytokines IL-1 $\beta$ and IL-1 $8^{17,22,26-29}$.

Majeed syndrome is an autosomal recessive disorder that is characterized with early-onset CRMO, Sweet syndrome-like skin rash, dyserythropoietic anemia, and joint contractures. It is caused by the loss of function mutations in the LPIN2 gene which encodes phosphatase lpin2, a phosphatidate phosphatase that plays a role in lipid metabolism, thus resulting in the overactivation of TLR4 pathways and systemic inflammation. Lipin2-deficient monocytes produce high amounts of pro-inflammatory cytokines IL-6 and tumor necrosis factor alpha (TNF- $\alpha$ ) when stimulated by saturated fatty acids. The overexpression of LPIN2 on the other hand reduces inflammatory cytokine levels ${ }^{22}$. For Majeed syndrome, there is evidence that it is an IL-1 $\beta$-mediated disease, since bone inflammation and serum inflammation markers improve in response to an IL- $1 \beta$ blockade, while TNF- $\alpha$ blockers have almost no effect ${ }^{26}$. Therefore, nonsteroidal anti-inflammatory drugs (NSAIDs), corticosteroids, and IL-1-blocking agents should be considered as empirical treatment.

DIRA is an autosomal recessive mutation in the ILIRN gene, encoding for the IL-1 receptor antagonist (IL-1RA), a post-translational regulator of IL-1 signaling resulting in uncontrolled proinflammatory cytokine and chemokine expression. DIRA is characterized by early-onset oral mucosal ulceration, pustular rash, rib widening with periostitis, multifocal osteolytic lesions, and heterotopic ossification. DIRA leads to a severe systemic inflammatory response syndrome and respiratory failure ${ }^{17}$ due to the lack of functional IL-1RAs and subsequently uncontrolled IL- $1 \beta$ signaling. Recombinant IL-1RAs therapy is known for therapeutic option ${ }^{17,27}$.

PAPA is an autosomal dominant mutation in the PSTPIP1 gene which binds to pyrin and regulates the actin cytoskeleton. Pyrin is a central negative regulator of the NLRP3 inflammasome by releasing of IL-1 $\beta$. PAPA is characterized by pyoderma gangrenosum-like ulcerative lesions, cystic acne and sterile erosive arthritis. Therapeutic options involve local and/or systemic steroids, thalidomide, cyclosporine, tacroli- mus, intravenous immunoglobulin, TNF- $\alpha$ blockers, and IL-1 blocking agents $^{28,29}$.

\section{CNO/CRMO Studies}

$\mathrm{CNO}$ in the jaw presents with mild or non-specific clinical symptoms, which is not recognized by patients and medical staff resulting in a delayed final diagnosis. Although CNO is associated with other general syndromes or genetic diseases including SAPHO, Majeed syndrome, DIRA, and PAPA, a genetic or molecular basis for animal studies have been provoked and found to be associated with other autoinflammatory conditions such as IBD, acne, ankylosing spondylitis, and psoriasis.

\section{Microbiome in $\mathrm{CNO} / \mathrm{CRMO}$}

A microbiota, microbiome, is the collective genomes of commensal or pathogenic microorganisms in plants or animals. Disturbances to the microbiome, particularly of the gut, have been proven to contribute to altered immune cell distribution and inflammatory phenotypes in several diseases including immunologic, hormonal, and metabolic disorders ${ }^{30}$. In rheumatoid arthritis, the replacement of Lactobacillus spp. with Prevotella spp. has been linked to disease activity and outcomes. Liao et al. ${ }^{31}$ published the first study that found that disturbances in the gut microbiome may contribute to sterile bone inflammation.

In humans, it could be proposed a potential pathophysiological connection of an altered skin microbiome and aseptic bone inflammation in $\mathrm{CNO}$, because both of severe cystic acne and $\mathrm{CNO}$, share the imbalanced expression and secretion of proinflammatory antiinflammatory cytokines ${ }^{32-34}$.

\section{Murine models of CRMO}

Two well-characterized murine models have been developed, chronic multifocal osteomyelitis ( $\mathrm{cmo}$ ) mice carrying a spontaneous homozygous mutation (p.L98P) and lupo mice carrying a chemically-induced homozygous mutation (p.I282N) in the Pstpip2 gene ${ }^{35,36}$. Two models could be developed to cytokine and chemokine dysregulation, systemic inflammation, extramedullary hematopoiesis, skin inflammation, and sterile osteomyelitis. As in the case of CRMO patients, cmo mice exhibit a central involvement of IL-1 $\beta$ in disease pathophysiology and the complete deficiency of Pstpip 2 gene results in the dysregulated production of IL-1 $\beta$ by 
neutrophils and enhanced osteoclastogenesis ${ }^{35}$.

\section{Chemokine and cytokine dysregulation in $\mathrm{CNO} /$ CRMO}

The molecular mechanisms of $\mathrm{CNO} / \mathrm{CRMO}$ have been suggested as many different pathways and hypotheses, inflammasome and consequent imbalance between proinflammatory and anti-inflammatory cytokines.

TLR4/MAPK/inflammasome signaling pathway could contribute to the inflammatory phenotype in CRMO, inflammatory bone loss and synovial inflammation in IL-10-deficient mice were linked to increased NOD-like receptor protein 3 (NLRP3) inflammasome activation ${ }^{37,38}$. This activation will increase mRNA expression of inflammasome including ASC, NLRP3, and caspase-1, as well as increased IL- $1 \beta$ transcription and release from monocytes of CRMO patients ${ }^{37,38}$. An immunomodulatory reversible function by IL-10 and IL-19 on enhanced inflammasome activation with IL-1 $\beta$ secretion could be suggested ${ }^{23}$.

The imbalanced expression of antiinflammtory cytokines such as IL-10 and IL-19, and pro-inflammatory cytokines such as IL-1, IL-6, TNF- $\alpha$, and IL-20, may result in increased osteoclast differentiation and activation through enhanced interactions between the receptor activator of nuclear factor$\kappa \mathrm{B}$ (RANK) and its soluble ligand RANKL on osteoclast precursor cells ${ }^{19,33}$. IL-10 expression is predetermined by genetic variants within the IL-10 proximal promoter region, by blocking encoding for high IL-10 expression (GCC) ${ }^{27}$, which is one of clue for the IL-10 medication to the CRMO patients.

Additionally, a CRMO susceptibility gene has been identified through whole exome sequencing and gene expression microarrays. FBLIM1 has been suggested to act as an antiinflammatory molecule that controls bone remodeling through the regulation of RANKL activation through ERK1/2 phosphorylation. On the transcriptional level, FBLIM1 expression is regulated by the transcription factor STAT3. Since the immune regulatory cytokine IL-10 induces STAT3 activation, the aforementioned haplotype blocks within the IL-10 promoter may be involved in the pathophysiology of CNO. Cox et al. ${ }^{39}$ demonstrated that IL-10 promoter haplotypes may contribute to reduced STAT3 activation. Although preliminary results regarding the kinetics of innate and adaptive inflammatory responses in $\mathrm{CNO} / \mathrm{CRMO}$, outline for future targeted medications should be continued based on the specific expressions of cytokines and chemokines ${ }^{19,40}$.

\section{Treatment and Biomarkers for CNO/CRMO in the Jaw}

The treatment of patients with CNO/CRMO in the jaw should not always be through surgical means and that medications should be considered. Only small portions of bone biopsies including both cortical and marrow bony architecture could be confirmed for the exclusion of any bacterial osteomyelitis. NSAIDs are representative medication drugs for $\mathrm{CNO} / \mathrm{CRMO}$. Other recently approved medications include corticosteroids, bisphosphonates and disease-modifying antirheumatic drugs (DMARDs) including methotrexate, sulfasalazine and anti-TNF agents.(Fig. 3)

\section{Biomarkers for $\mathrm{CNO}$ diagnosis and monitoring}

Sstandardized and accepted parameters have not yet to be reported despite many basic studies and follow-up clinical studies regarding $\mathrm{CNO} / \mathrm{CRMO}$ biomarkers. Known classical inflammation markers, such as CRP and ESR, are not specific. Therefore, in unclear cases, bone biopsies must be considered as the first action prior to prescribing any medications $^{32}$.

A clinical score for CNO patients was developed by Jansson et al. ${ }^{41}$, and Beck et al. ${ }^{42}$ presented the Pediatric CNO (PedCNO) score, which includes 5 measurements such as ESR, number of radiological lesions, severity of disease estimated by the physician, severity of disease estimated by

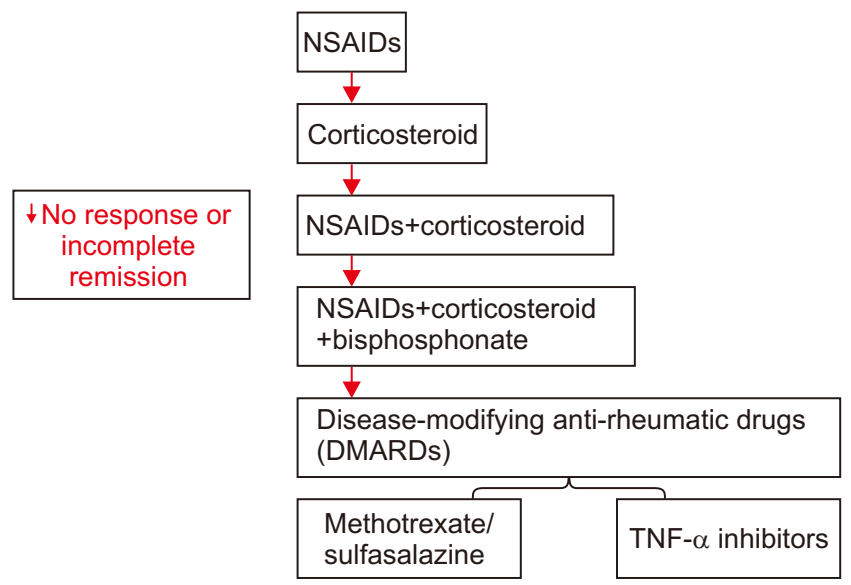

Fig. 3. Schematic diagrams for the generalized medication protocols of CNO/CRMO in the jaw. (CNO: chronic non-bacterial osteomyelitis, CRMO: chronic recurrent multifocal osteomyelitis, NSAIDs: nonsteroidal anti-inflammatory drugs, TNF- $\alpha$ : tumor necrosis factor alpha)

Soung Min Kim et al: Chronic non-bacterial osteomyelitis in the jaw. J Korean Assoc Oral Maxillofac Surg 2019 
the patient or parent, and the Childhood Health Assessment Questionnaire. However, the clinical score has not been considered for other ABD involving the jaw, metabolic disorders, lymphoma, leukemia, LCH, and mycobacterial bone infections. El-Shanti and Ferguson ${ }^{11}$ identified a set of nine serum inflammation markers including IL-6, IL-12, MIG, RANTES, IL-1RA, soluble IL-2 receptor (sIL-2R), monocyte chemotactic protein-1 (MCP-1), macrophage inflammatory protein$1 \mathrm{~b}$ (MIP-1b) and mast cell derived chemokine eotaxin. These serum protein arrays may be used for the diagnosis of $\mathrm{CNO}$ / CRMO, but larger multi-institute cohort studies should be performed.

Unbalanced cytokine and chemokine expression could be used for the diagnosis of $\mathrm{CNO} / \mathrm{CRMO}^{19,38,43}$. Molecular works of monocytes from CRMO patients showed decreased levels of IL-10, IL-1RA and granulocyte monocyte colonystimulating factor, and showed increased levels of IL-1 $\beta$, IL6 , TNF- $\alpha$, IL-8, Interferon gamma-induced protein 10, MCP1, MIG, MIP-1a, and MIP- $1 \mathrm{~b}^{38,43}$. And the increased levels of the NLRP3 from inflammatory infiltrates of CNO/CRMO could be translated into IL-1 $\beta$ and IL-10 protein expression in bacterial osteomyelitis. This NLRP3 component in the gene expression profiles would be applicated in the $\mathrm{CNO} / \mathrm{CRMO}$ in jaw cases.

Not like in the bacterial osteomyelitis, predominant neutrophils and monocytes are usually moderately infiltrated with bone marrow fibrosis in the CNO/CRMO bone biopsy ${ }^{32,33}$.

\section{Treatments of $\mathrm{CNO}$ in the jaw}

Any pathological involvement of the jaw should be managed with medicinal approaches at first and not with surgical considerations. Usually, NSAIDs, corticosteroids, and DMARDs such as methotrexate or sulfasalazine, could be considered for general CNO/CRMO management. Anti-TNF agents including proinflammatory cytokines and bisphosphonates (BPs) could be additionally considered.(Fig. 3)

NSAIDs are the first-line treatment of choice for the treatment of initial $\mathrm{CNO} / \mathrm{CRMO}$ in jaw patients with the most common choice being 10 to $15 \mathrm{mg} / \mathrm{kg} /$ day of naproxen in 2 divided doses which is effective in $70 \%$ of patients ${ }^{9}$. NSAIDs are effective in approximately two-thirds of $\mathrm{CNO} / \mathrm{CRMO}$ patients, and NSAIDs are more effective in $\mathrm{CNO}$ patients compared with only CRMO patients ${ }^{24}$. And another data showed that NSAIDs did not induce true real remission in CNO patients with a clinically asymptomatic state in $43 \%$ after 6 months and in $51 \%$ at 12 months, and also NSAIDs may not be sufficient for long-term remission in the majority of patients ${ }^{9,42}$.

For the management of CRMO in jaw patients, definitely NSAIDs should be considered as the first medication only excluding the relapsed or no completed remission cases. $\mathrm{Cy}-$ clooxygenases inhibition is activated by NSAIDs for the conversion of arachidonic acid into prostaglandins, which is essential procedure of osteoclast activation in jaw ${ }^{3}$. Jaw edema or any kinds of structural damage with NSAIDs should be followed-up periodically.

Widely accepted treatment consensus remains empirical, despite many collected case series of NSAID medications, measurements of disease activity, treatment goal definitions, and outcome measurements. Corticosteroids in $\mathrm{CNO} / \mathrm{CRMO}$ management could be recommended as an intermediately between failed NSAIDs and other drugs initiation. Oral glucocorticoid medication is recommended as $2 \mathrm{mg}$ prednisone/ $\mathrm{kg}$ /day for 1 week and tapered discontinuation by $25 \%$ every 5 days ${ }^{44}$. Intravenous steroid infusion could be effective in selected recurrent patients but a standardized medication protocol is not known yet ${ }^{45}$. The inhibition pathway of phospholipase A2 is well known for reduced prostaglandin levels as the corticosteroids mechanisms. In the case of $\mathrm{CNO} / \mathrm{CRMO}$ patients, the inhibition of nuclear factor- $\kappa \mathrm{B}$-dependent gene activation could be also useful for the reduced proinflammatory cytokine levels such as IL- 6 and the TNF- $\alpha$ gene ${ }^{39}$.

In spite of its quick control of inflammatory activity, longterm effects had not been expected in $\mathrm{CNO} / \mathrm{CRMO}$ patients. The effective management with bisphosphonate or anti-TNF agents have been reported to be highly effective for the longlasting remission ${ }^{12,33,42}$. The representative osteoclast inhibition drug, BPs, could increase bone mass with improved mineralization in the postmenopausal and glucocorticoidinduced osteoporosis. Although BPs have severe side effects such as BRONJ, this is effective in $\mathrm{CNO} / \mathrm{CRMO}$ patients by modify pains through suppression of proinflammatory cytokines, such as TNF- $\alpha$, IL- 6 , and IL- $1^{46}$. BPs could be recommended in no effective cases of NSAIDs, corticosteroids, or DMARDs. Among BPs, pamidronate could be first recommended in the refractory pediatric $\mathrm{CNO} / \mathrm{CRMO}$ patients, which could be administered as an initial dose of $0.5 \mathrm{mg} / \mathrm{kg} /$ day followed by $1 \mathrm{mg}$ on days 2 and $3 \mathrm{mg}$ every 3 months, or 1 dose of $1 \mathrm{mg} / \mathrm{kg}$ every month for 1 to 6 months $\mathrm{s}^{32,47-49}$.

Due to its exact pathogenesis is not revealed, any molecules during having its role in a little bit known sequences could be blocked for the additional treatment option in $\mathrm{CNO}$ / CRMO patients. Methotrexate and sulfasalazine are known 
to reduce inflammatory cytokine expression in recalcitrant $\mathrm{CNO} / \mathrm{CRMO}$ patients and are more commonly used in North America and Europe ${ }^{1}$. More recently, DMARDs may be used more and more due to several side effects or no complete remission of NSAIDs especially in CRMO in jaw ${ }^{24}$.

Since IL-1 and TNF- $\alpha$ are generally important in the inflammatory pathogenesis, IL-1 blocking agents and TNF- $\alpha$ inhibitors can be also considered in CNO/CRMO treatment. TNF inhibitors could also be used with NSAIDs and corticosteroids for specifically blocking TNF- $\alpha$ and for restoring the balance between this highly potent proinflammatory cytokine and immune modulatory cytokines IL-10 and IL-19 $9^{23-25,27}$. Although there is no reliable report, RANKL blockade with denosumab, which is the recombinant RANK ligand inhibitor, may reduce osteoclast activation and inflammatory bone loss in CRMO in jaw. These functions are deduced from the differentiation and activation of osteoclasts, TNF- $\alpha$ versus IL-10 and IL-19, which are counteracting mediators ${ }^{50}$.

Recently, biomarkers including IL-12, MCP-1, and sIL$2 \mathrm{R}$ were introduced for acting of treatment response to NSAIDs ${ }^{51,52}$. Further evaluation of these medications, such as IL-12, MCP-1, and sIL-2R, could be used as the progression of $\mathrm{CNO} / \mathrm{CRMO}$ in jaw.

\section{Conclusion}

CRMO in jaw must be diagnosed with the differentiation of chronic bacterial osteomyelitis, and a bone biopsy is essential. The early diagnosis of CRMO in jaw will prevent unnecessary and prolonged antibiotic usage and unnecessary surgical intervention. The updated researches of several pathophysiological alterations in $\mathrm{CNO} / \mathrm{CRMO}$ should be studied more for its exact causes and allow for the creation of target-directed treatment options.

\section{ORCID}

Soung Min Kim, https://orcid.org/0000-0002-6916-0489

Suk Keun Lee, https://orcid.org/0000-0001-6255-4197

\section{Authors' Contributions}

S.M.K. designed this manuscript and wrote, S.K.L. perfomed related research works and advised. Both authors read and approved the final manuscript.

\section{Acknowledgements}

This work was supported by Research Resettlement Fund for the new faculty of Seoul National University, and by a grant of the Korean Health Technology R\&D Project, Ministry of Health \& Welfare, Republic of Korea (HI15C0689).

\section{Conflict of Interest}

No potential conflict of interest relevant to this article was reported.

\section{References}

1. Hofmann SR, Schnabel A, Rösen-Wolff A, Morbach H, Girschick HJ, Hedrich CM. Chronic nonbacterial osteomyelitis: pathophysiological concepts and current treatment strategies. J Rheumatol 2016;43:1956-64.

2. Hofmann SR, Kapplusch F, Girschick HJ, Morbach H, Pablik J, Ferguson PJ, et al. Chronic recurrent multifocal osteomyelitis (CRMO): presentation, pathogenesis, and treatment. Curr Osteoporos Rep 2017;15:542-4.

3. Giedion A, Holthusen W, Masel LF, Vischer D. Subacute and chronic "symmetrical" osteomyelitis. Ann Radiol (Paris) 1972;15:329-42.

4. Andersson R. Effective treatment with interferon-alpha in chronic recurrent multifocal osteomyelitis. J Interferon Cytokine Res 1995;15:837-8.

5. Wang Y, Yang C, Zhang W, Lu Y, Wei W, Han Z, et al. Monofocal chronic nonbacterial osteomyelitis in the mandible accompanied with mucocutaneous disease. J Craniofac Surg 2017;28:e547-51.

6. Padwa BL, Dentino K, Robson CD, Woo SB, Kurek K, Resnick $\mathrm{CM}$. Pediatric chronic nonbacterial osteomyelitis of the jaw: clini$\mathrm{cal}$, radiographic, and histopathologic features. J Oral Maxillofac Surg 2016;74:2393-402.

7. Monsour PA, Dalton JB. Chronic recurrent multifocal osteomyelitis involving the mandible: case reports and review of the literature. Dentomaxillofac Radiol 2010;39:184-90.

8. Hedrich CM, Hahn G, Girschick HJ, Morbach H. A clinical and pathomechanistic profile of chronic nonbacterial osteomyelitis/ chronic recurrent multifocal osteomyelitis and challenges facing the field. Expert Rev Clin Immunol 2013;9:845-54.

9. Hedrich CM, Hofmann SR, Pablik J, Morbach H, Girschick HJ. Autoinflammatory bone disorders with special focus on chronic recurrent multifocal osteomyelitis (CRMO). Pediatr Rheumatol Online J 2013;11:47.

10. Stern SM, Ferguson PJ. Autoinflammatory bone diseases. Rheum Dis Clin North Am 2013;39:735-49.

11. El-Shanti HI, Ferguson PJ. Chronic recurrent multifocal osteomyelitis: a concise review and genetic update. Clin Orthop Relat Res 2007;462:11-9.

12. Borzutzky A, Stern S, Reiff A, Zurakowski D, Steinberg EA, Dedeoglu F, et al. Pediatric chronic nonbacterial osteomyelitis. Pediatrics 2012;130:e1190-7.

13. Hofmann C, Wurm M, Schwarz T, Neubauer H, Beer M, Girschick $\mathrm{H}$, et al. A standardized clinical and radiological follow-up of patients with chronic non-bacterial osteomyelitis treated with pamidronate. Clin Exp Rheumatol 2014;32:604-9.

14. Khanna G, Sato TS, Ferguson P. Imaging of chronic recurrent multifocal osteomyelitis. Radiographics 2009;29:1159-77.

15. Jurik AG, Egund N. MRI in chronic recurrent multifocal osteomy- 
elitis. Skelet Radiol 1997;26:230-8.

16. Morbach H, Schneider P, Schwarz T, Hofmann C, Raab P, Neubauer $\mathrm{H}$, et al. Comparison of magnetic resonance imaging and 99mTechnetium-labelled methylene diphosphonate bone scintigraphy in the initial assessment of chronic non-bacterial osteomyelitis of childhood and adolescents. Clin Exp Rheumatol 2012;30:57882.

17. Beck C, Girschick HJ, Morbach H, Schwarz T, Yimam T, Frenkel $\mathrm{J}$, et al. Mutation screening of the IL-1 receptor antagonist gene in chronic non-bacterial osteomyelitis of childhood and adolescence. Clin Exp Rheumatol 2011;29:1040-3.

18. Golla A, Jansson A, Ramser J, Hellebrand H, Zahn R, Meitinger $\mathrm{T}$, et al. Chronic recurrent multifocal osteomyelitis (CRMO): evidence for a susceptibility gene located on chromosome 18q21.318q22. Eur J Hum Genet 2002;10:217-21.

19. Nakashima T, Takayanagi H. Osteoimmunology: crosstalk between the immune and bone systems. J Clin Immunol 2009;29:555-67.

20. Nakashima T, Takayanagi H. Osteoclasts and the immune system. J Bone Miner Metab 2009;27:519-29.

21. Sharma M, Ferguson PJ. Autoinflammatory bone disorders: update on immunologic abnormalities and clues about possible triggers. Curr Opin Rheumatol 2013;25:658-64.

22. Hamel J, Paul D, Gahr M, Hedrich CM. Pilot study: possible association of IL10 promoter polymorphisms with CRMO. Rheumatol Int 2012;32:555-6.

23. Hofmann SR, Kubasch AS, Ioannidis C, Rösen-Wolff A, Girschick $\mathrm{HJ}$, Morbach H, et al. Altered expression of IL-10 family cytokines in monocytes from CRMO patients result in enhanced IL-1 $\beta$ expression and release. Clin Immunol 2015;161:300-7.

24. Hofmann SR, Morbach H, Schwarz T, Rösen-Wolff A, Girschick HJ, Hedrich CM. Attenuated TLR4/MAPK signaling in monocytes from patients with CRMO results in impaired IL-10 expression. Clin Immunol 2012;145:69-76.

25. Hofmann SR, Schwarz T, Möller JC, Morbach H, Schnabel A, Rösen-Wolff A, et al. Chronic non-bacterial osteomyelitis is associated with impaired Sp1 signaling, reduced IL10 promoter phosphorylation, and reduced myeloid IL-10 expression. Clin Immunol 2011;141:317-27.

26. Hedrich CM, Bream JH. Cell type-specific regulation of IL-10 expression in inflammation and disease. Immunol Res 2010;47:185206.

27. Hofmann SR, Rösen-Wolff A, Tsokos GC, Hedrich CM. Biological properties and regulation of IL-10 related cytokines and their contribution to autoimmune disease and tissue injury. Clin Immunol 2012;143:116-27.

28. Lindor NM, Arsenault TM, Solomon H, Seidman CE, McEvoy MT. A new autosomal dominant disorder of pyogenic sterile arthritis, pyoderma gangrenosum, and acne: PAPA syndrome. Mayo Clin Proc 1997;72:611-5.

29. Smith EJ, Allantaz F, Bennett L, Zhang D, Gao X, Wood G, et al. Clinical, molecular, and genetic characteristics of PAPA syndrome: a review. Curr Genomics 2010;11:519-27.

30. Shreiner AB, Kao JY, Young VB. The gut microbiome in health and in disease. Curr Opin Gastroenterol 2015;31:69-75.

31. Liao HJ, Chyuan IT, Wu CS, Lin SW, Chen KH, Tsai HF, et al. Increased neutrophil infiltration, IL-1 production and a SAPHO syndrome-like phenotype in PSTPIP2-deficient mice. Rheumatology (Oxford) 2015;54:1317-26.

32. Schnabel A, Range U, Hahn G, Siepmann T, Berner R, Hedrich $\mathrm{CM}$. Unexpectedly high incidences of chronic non-bacterial as compared to bacterial osteomyelitis in children. Rheumatol Int 2016;36:1737-45.

33. Morbach H, Hedrich CM, Beer M, Girschick HJ. Autoinflammatory bone disorders. Clin Immunol 2013;147:185-96.
34. Cassel SL, Janczy JR, Bing X, Wilson SP, Olivier AK, Otero JE, et al. Inflammasome-independent IL- $1 \beta$ mediates autoinflammatory disease in Pstpip2-deficient mice. Proc Natl Acad Sci U S A 2014;111:1072-7.

35. Chitu V, Nacu V, Charles JF, Henne WM, McMahon HT, Nandi S, et al. PSTPIP2 deficiency in mice causes osteopenia and increased differentiation of multipotent myeloid precursors into osteoclasts. Blood 2012;120:3126-35.

36. Grosse J, Chitu V, Marquardt A, Hanke P, Schmittwolf C, Zeitlmann L, et al. Mutation of mouse Mayp/Pstpip2 causes a macrophage autoinflammatory disease. Blood 2006;107:3350-8.

37. Greenhill CJ, Jones GW, Nowell MA, Newton Z, Harvey AK, Moideen AN, et al. Interleukin-10 regulates the inflammasomedriven augmentation of inflammatory arthritis and joint destruction. Arthritis Res Ther 2014;16:419.

38. Scianaro R, Insalaco A, Bracci Laudiero L, De Vito R, Pezzullo $\mathrm{M}$, Teti A, et al. Deregulation of the IL-1 $\beta$ axis in chronic recurrent multifocal osteomyelitis. Pediatr Rheumatol Online J 2014;12:30.

39. Cox AJ, Darbro BW, Laxer RM, Velez G, Bing X, Finer AL, et al. Recessive coding and regulatory mutations in FBLIM1 underlie the pathogenesis of chronic recurrent multifocal osteomyelitis (CRMO). PLoS One 2017;12:e0169687.

40. Ferguson PJ, El-Shanti HI. Autoinflammatory bone disorders. Curr Opin Rheumatol 2007;19:492-8.

41. Jansson AF, Müller TH, Gliera L, Ankerst DP, Wintergerst U, Belohradsky BH, et al. Clinical score for nonbacterial osteitis in children and adults. Arthritis Rheum 2009;60:1152-9.

42. Beck C, Morbach H, Beer M, Stenzel M, Tappe D, Gattenlöhner S, et al. Chronic nonbacterial osteomyelitis in childhood: prospective follow-up during the first year of anti-inflammatory treatment. Arthritis Res Ther 2010;12:R74.

43. Boehncke WH, Schön MP. Psoriasis. Lancet 2015;386:983-94.

44. Girschick HJ, Zimmer C, Klaus G, Darge K, Dick A, Morbach H. Chronic recurrent multifocal osteomyelitis: what is it and how should it be treated? Nat Clin Pract Rheumatol 2007;3:733-8.

45. Holden W, David J. Chronic recurrent multifocal osteomyelitis: two cases of sacral disease responsive to corticosteroids. Clin Infect Dis 2005;40:616-9.

46. Miettunen PM, Wei X, Kaura D, Reslan WA, Aguirre AN, Kellner JD. Dramatic pain relief and resolution of bone inflammation following pamidronate in 9 pediatric patients with persistent chronic recurrent multifocal osteomyelitis (CRMO). Pediatr Rheumatol Online J 2009; 7:2.

47. Compeyrot-Lacassagne S, Rosenberg AM, Babyn P, Laxer RM. Pamidronate treatment of chronic noninfectious inflammatory lesions of the mandible in children. J Rheumatol 2007;34:1585-9.

48. Eleftheriou D, Gerschman T, Sebire N, Woo P, Pilkington CA, Brogan PA. Biologic therapy in refractory chronic non-bacterial osteomyelitis of childhood. Rheumatology (Oxford) 2010;49:150512.

49. Schnabel A, Range U, Hahn G, Berner R, Hedrich CM. Treatment response and longterm outcomes in children with chronic nonbacterial osteomyelitis. J Rheumatol 2017;44:1058-65.

50. Herlin T, Fiirgaard B, Bjerre M, Kerndrup G, Hasle H, Bing X, et al. Efficacy of anti-IL-1 treatment in Majeed syndrome. Ann Rheum Dis 2013;72:410-3.

51. Hofmann SR, Kubasch AS, Range U, Laass MW, Morbach H, Girschick HJ, et al. Serum biomarkers for the diagnosis and monitoring of chronic recurrent multifocal osteomyelitis (CRMO). Rheumatol Int 2016;36:769-79.

52. Miettunen PMH. Chronic non-bacterial osteitis/chronic recurrent multifocal osteomyelitis. In: Baptista MS, Tardivo JP, eds. Osteomyelitis. London: InTech; 2012:89-118. 